\title{
Novedades sobre la distribución del macroendemismo bético Castrilanthemum debeauxii (Degen, Hervier \& É. Rev.) Vogt \& Oberprieler
}

\author{
Jesús del Río ${ }^{1}$ \& Leonardo Gutiérrez ${ }^{2}$ \\ 1 Departamento de Geodiversidad y Biodiversidad. Delegación Territorial de Agricultura, Pesca y Medio Ambiente de Granada. \\ Junta de Andalucía. Av. Joaquina Egüaras, 2. 18013 Granada. \\ 2 Jardín Botánico Umbría de la Virgen. Red Andaluza de Jardines Botánicos y Micológico. Consejería de Medio Ambiente y \\ Ordenación del Territorio. Junta de Andalucía. Paraje de la Umbría, s/n. 04838 María (Almería).
}

\section{Correspondencia}

J. del Río

E-mail: jesus.rio@juntadeandalucia.es

TIf.:+34 600145043

Recibido: 29 octubre 2013

Aceptado: 14 noviembre 2013

Publicado on-line: 25 noviembre 2013

\section{Resumen}

Se presentan nuevos datos sobre el área de distribución del raro macroendemismo Castrilanthemum debeauxii, confirmando su presencia en las provincias de Jaén y Granada y aportando como novedad su presencia en la provincia de Albacete.

Palabras clave: Compositae, área distribución, Albacete, Granada, Jaén.

\begin{abstract}
New data about the distribution of Baetic macroendemism Castrilanthemum debeauxii (Degen, Hervier \& É. Rev.) Vogt \& Oberprieler

We present new data on the distribution area of rare macroendemism Castrilanthemum debeauxii, confirming its presence in the provinces of Jaen and Granada and contributing as a new presence in the province of Albacete.
\end{abstract}

Key words: Compositae, Distribution area, Albacete, Granada, Jaen.

\section{Introducción}

Castrilanthemum debeauxii es una especie de la familia Compositae perteneciente a un genero monoespecífico descrito en 1996 (Vogt \& Oberprieler 1996). La especie fue descubierta en las sierras del nordeste de Granada y oeste de Jaén por Elysée Reverchon a principios del siglo XX, denominándose inicialmente Pyretrum debeauxii Degen, Hervier \& É Rev. (Hervier 1905). Las primeras reseñas y pliegos recolectados la situaban en diversas sierras de Jaén que colindan con la provincia de Granada, como son la Sierra de la Cabrilla, la vertiente jienense de la Sierra de Castril y una zona denominada Sierra del Cuarto (Hervier 1905, 1906), que debe corresponderse con una zona montañosa de Santiago de la Espada, contigua a la cabecera del río Castril, al nordeste de Sierra de Castril y Sierra Seca (Lacaita 1929). Posteriormente no se conocieron nuevas referencias de su presencia hasta 1978, año en el que fue colectada en la Sierra de la Guillimona, dentro de la provincia de Granada (Leal et al. 1980). Ya a principios del siglo XXI, con motivo del proyecto AFA (Atlas de Flora Amenazada), se realizaron diversas prospecciones detalladas en las zonas donde fue descubierta, pero tan solo pudo localizarse en la Sierra de la Guillimona (Blanca et al. 2004).

Durante la campaña de 2013 se ha vuelto a realizar una prospección detallada por su área de distribución, habiéndose localizado, además de en la Sierra de la Guillimona (Granada), en una zona montañosa de Santiago de la Espada (Jaén) que podría corresponderse con lo que Hervier (1906) 
denominaba "Sierra del Cuarto" y, lo que es más interesante, también se ha hallado en la Sierra de la Hoya del Espino, en la provincia de Albacete. Además, se ha verificado la presencia discontinua de $C$. debeauxii en todo el eje de la Sierra de la Guillimona desde el extremo oriental, en el Puerto del Pinar, hasta el occidental, en el Puerto de la Losa, en una banda situada entre los 1.600-1.900 msnm y preferentemente en orientaciones con componente oeste. No ha podido localizarse en las localidades de los pliegos originales como son Sierra de Castril y Sierra de la Cabrilla, y aunque no se descarta su presencia en la actualidad, también es cierto que la fuerte presión ganadera que se ha detectado en estos territorios hace muy difícil la existencia de grandes núcleos de población.

\section{Resultados}

Los nuevos núcleos poblacionales encontrados representan la continuación del eje de distribución conocido a lo largo de la vertiente norte de la Sie- rra de la Guillimona (Figura 1). Los datos de localización más significativos, depositados en el Herbario de la Universidad de Almería (HUAL), son: los siguientes:

Albacete: Nerpio, Sierra de la Hoya del Espino: collado de Melgares, 30SWH4714, $1.750 \mathrm{msnm}$, 13-VI-2013, L. Gutiérrez, HUAL 24954. Granada: Huéscar, Sierra de los Cuartos: Malamujer, próximo a la Cañada de la Hunguilla, 30SWH3507, 1.700 msnm, 13-VI-2013, L. Gutiérrez, HUAL 24956. La Puebla de Don Fadrique, Sierra de la Guillimona: al suroeste del Puerto del Pinar, Camino de los Leñadores, 30SWH4410, 1.710 msnm, 13-VI-2013, L. Gutiérrez, HUAL 24955. Jaén: Santiago-Pontones, Sierra de los Cuartos: El Tejuelo, próximo a La Manga, 30SWH3406, 1.670 msnm, 13-VI-2013, L. Gutiérrez, HUAL 24957.

Todos ellos aparecen formando parte de la vegetación de piornales sobre suelos calizos pedregosos de montaña, pertenecientes al orden Erinacetalia anthyllidis Quézel 1951 y en el límite infe-

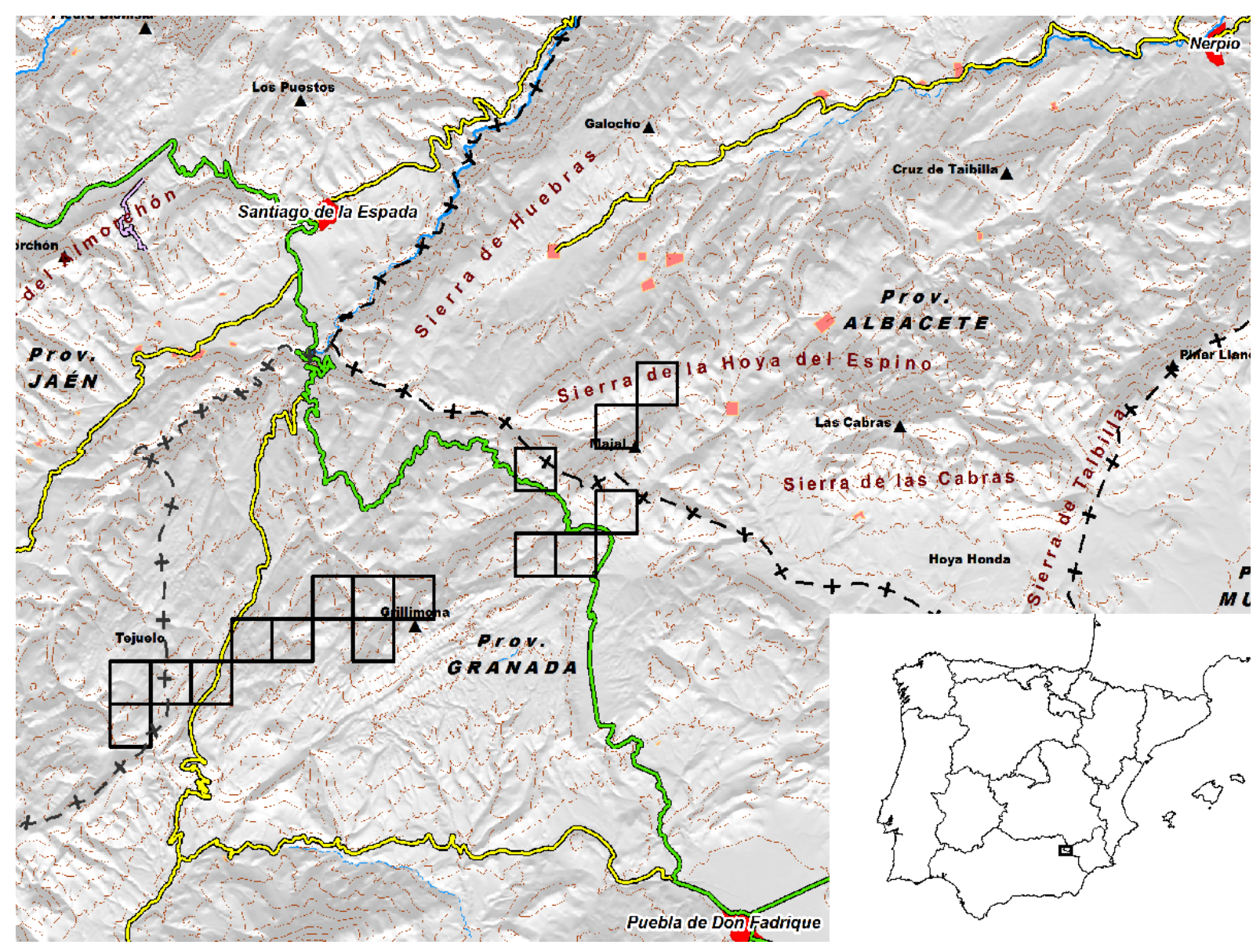

Figura 1: Mapa de distribución conocida de Castrilanthemum debeauxii en cuadriculas UTM 1x1 km.

Figure 1: Map of known distribution of Castrilanthemum debeauxii in 1x1 km UTM squares. 
rior del dominio de la serie oromediterránea bética basófila de Juniperus sabina (Daphno oleoidi-Pineto sylvestris $\mathrm{S}$.). Se sitúan en la transición de los pisos Supra-Oromediterráneo, con ombroclima Subhúmedo, y preferencia por las orientaciones con componente oeste.

Su condición de especie anual de clima mediterráneo conlleva fuertes fluctuaciones demográficas interanuales en sus efectivos, en función de las condiciones meteorológicas concretas de cada año. Este factor, junto con su parecido con Anthemis tuberculata, su pequeño tamaño y corta fenología, explicaría el que frecuentemente haya pasado desapercibida. Su sensibilidad a las condiciones microclimáticas hace que su área de distribución no sea continua, observándose cierta predilección por las vertientes orientadas al oeste o noroeste. El hábitat óptimo parece corresponder con el piornal supra-oromediterráneo sobre laderas más o menos pedregosas con suelos poco desarrollados. Dentro de su área de distribución suele aparecer formando pequeños parches o mosaicos discontinuos, probablemente a consecuencia de su pobre capacidad de dispersión. Muestra cierta sensibilidad hacia el sobrepastoreo y el pisoteo excesivo producido por la ganadería. En aquellos lugares vallados y excluidos a la herbivoría, o con temporalidad de la misma, muestra mayor probabilidad de presencia.

Actualmente, toda su área de distribución potencial y conocida cae dentro de espacios naturales protegidos, ya sea con la figura de Parque Natural (P.N. Sierra de Castril y P.N. Sierras de Cazorla, Segura y Las Villas) o como Lugar de Importancia Comunitaria (LIC Sierras del Nordeste y LIC Sierras de Alcaraz y de Segura y Cañones del Segura y del Mundo). A pesar de ello, se considera un taxon amenazado y actualmente protegido por la legislación andaluza dentro de la categoría "En Peligro", por lo que se sugiere el mismo tratamiento para la legislación castellano-manchega. El hallazgo de estos nuevos núcleos demográficos mejora el diagnóstico sobre su estado de conservación; sin embargo, la reevaluación de su categoría de amenaza con los actuales criterios de la UICN (2012) no supone una rebaja de la categoría CR (En Peligro Crítico). El análisis con los nuevos datos nos lleva a clasificarla como CR B1b(iii)c(iv), puesto que se ha ampliado notablemente el número de localidades y por consiguiente el área de ocupación.
Con el fin de lograr un mejor entendimiento de las causas de amenaza que se ciernen sobre esta especie, investigadores del Real Jardín Botánico de Madrid están analizando su diversidad genética y caracterizando su biología reproductiva. Su interés estriba en que pudiera tratarse de un fósil vivo, taxonómicamente aislado, que forma parte de los últimos supervivientes de linajes evolutivos antiguos (Vargas 2010). Algunos resultados preliminares de estos estudios podrían indicar una nueva amenaza proveniente de la depredación de sus semillas por parte de un insecto (Jiménez-Mejías et al. 2012).

\section{Agradecimientos}

A Isaac García y Demetrio Sánchez, Agentes de Medio Ambiente de la Junta de Andalucía por la aportación de buena parte de las nuevas referencias geográficas; y a Antonio Castillo, Técnico de la Delegación Territorial de Agricultura, Pesca y Medio Ambiente de Granada, por su ayuda en la confección del mapa de distribución.

\section{Referencias}

Blanca, G., Gutiérrez, L., Luque, P., Benavente, A., Morales, C. \& Garrrido A. 2004. Castrilanthemum debeaxii (Degen, Hervier et E. Rev) Vogt et Oberprieler in Bañares A., Blanca G., Güemes J., Moreno J.C. \& Ortiz S., eds. 2004. Atlas y Libro Rojo de la Flora Vascular Amenazada de España. Dirección General de Conservación de la Naturaleza. Madrid, 1.069 pp.

Hervier, J. 1905. Excursions botaniques de M. Elisée Reverchón dans le masif de la Sagra et à Vélez Rubio (Espagne) de 1898 a 1903. Bull. Acad. Inst. Geogr. Bot. 15 : 1-32, 89-120.

Hervier, J. 1906. Excursions botaniques de M. Elisée Reverchon dans le masif de la Sagra et à Vélez Rubio (Espagne) de 1898 a 1903. Bull. Acad. Inst. Geogr. Bot. 16 : 201-221.

Jiménez-Mejías, P., Amat, E., Alvarez, I. \& Vargas P. 2012. Nuevos nacimientos dan esperanza a las plantas españolas más amenazadas. Lychnos 9: 2227.

Lacaita, Ch. 1929. La Sierra de Cazorla et les excursions de M. Reverchon. Bull. Soc. Bot. Genève, ser. 2, 21: 120-134.

Leal, J., Ortiz, A., Pajarón, S. \& Rodriguez M.L. 1980. Números cromosómicos para la flora española 155161. Lagascalia 9: 269-272.

UICN. 2012. Categorías y Criterios de la Lista Roja de la UICN: Versión 3.1. Segunda edición. Gland, Suiza y Cambridge, Reino Unido: UICN. vi + 34pp. 
Vargas, P. 2010. Estudio de plantas amenazadas en España: ¿hay fósiles vivientes aún desconocidos?. Lychnos 3: 19-23.
Vogt, R. \& Oberprieler, Ch. 1996. Castrilanthemum Vogt \& Oberprieler, un nuevo género de Compositae-Anthemideae. Anales del Jardín Botánico de Madrid 54: 336-346. 